\title{
Placement Optimization of Reference Sensors for Indoor Tracking
}

\author{
Károly Farkas \\ Budapest University of Technology and Economics \\ Magyar tudósok krt. 2, H-1117 Budapest, Hungary \\ e-mail: farkask@hit.bme.hu
}

\begin{abstract}
Location-based applications and services are becoming widespread with the proliferation of smart mobile devices. They require position tracking which is still a challenge today in the indoor environment. Different wireless technologies and localization techniques can be used to obtain the position estimation. One possibility is deploying cheap ZigBee sensors as reference points and using triangulation to compute the position of the visiting node. This technique requires the reception of a wireless signal of at least three reference sensors with well-known positions everywhere within the covered area. In this paper, we propose OptiRef, our simulated annealing based method to find, in a given area, the optimal number and placement of the reference sensors to be used for indoor positioning. Our method has $O(n)$ complexity and shows linear run-time behavior. We investigate the performance of OptiRef via simulations focusing on ZigBee technology, although our method is generic and can be used with any kind of wireless technologies. The resulted reference point setup(s) can be considered as a good starting point for real environment design.
\end{abstract}

Keywords: Indoor localization; ZigBee; Triangulation; Simulated annealing; MATLAB

\section{Introduction}

Using some location-aware service is more and more common during our daily routines. Such services are based on location tracking. Collecting the location data is straightforward in open-air environments, e.g., via a GPS (Global Positioning System) receiver. However, indoor position tracking is a more challenging issue and still an actual research topic today. Several technologies and systems have been proposed and developed for indoor location sensing [1-3]. One possible approach is deploying cheap ZigBee (IEEE 802.15.4) sensors as reference points into the indoor area and using triangulation technics to derive the position of the visiting node. 
Triangulation methods estimate the target location based on the geometric properties of triangles [1]. They have two variants, such as lateration and angulation. Lateration derives the position by measuring the object's distances from multiple reference points. However, instead of measuring the distance directly some other characteristics are usually measured, such as received signal strengths (RSS), time of arrival (TOA) or time difference of arrival (TDOA). Then, the distance is derived by computing the attenuation of the emitted signal strength or by multiplying the radio signal velocity and the travel time. On the other hand, angulation locates an object by computing angles relative to multiple reference points. Regardless the applied variant of triangulation, the indispensable requirement for computing the location estimation is to receive the signal of at least three reference points everywhere within the given area.

In this paper, we investigate how to place wireless reference points (ZigBee sensors) to perceive the signal with strong enough strength of at least three sensors everywhere within the given indoor territory, but keep the number of deployed sensors as low as possible. Hence, the overall cost of the indoor positioning system and its operation expenses can be kept low. We propose OptiRef, a simulated annealing based algorithm to find the optimal number and placement of the reference points in a given area. Our method has $O(n)$ complexity and finds a solution, a good approximation of the global optimum, with linear run-time behavior. Furthermore, we have developed a simulation tool in the MATLAB [4] environment. We used this tool to implement OptiRef together with the ITU indoor wireless propagation model [5] simulating ZigBee signal propagation and to investigate the algorithm's behavior. Note, that our method is generic and can be used with any other wireless technologies (eg., Wi-Fi, Bluetooth, UWB). However, in this paper we focus our investigations on ZigBee sensors. Moreover, the reference point setup(s) given back by OptiRef can be considered as a good starting point for real environment design.

The rest of the paper is structured as follows. In Section 2, we briefly overview related approaches. OptiRef is proposed and described in Section 3, and we present its evaluation via simulations in Section 4. Finally, we give a short summary and sketch our future plans in Section 5.

\section{Related Work}

In the last years, several wireless technologies and systems have been proposed for indoor positioning [1-3]. One of them is the UWB (Ultra-Wide Band) technology which offers the highest accuracy, but it is quite an expensive solution still today. Cellular (mobile telephony) systems are also capable of providing positioning information, but their accuracy are low, and usually falls in the range of $50 \mathrm{~m}-100 \mathrm{~m}$, which is appropriate only for cell based positioning. 
On the other hand, most of the wireless positioning systems operate in the license free ISM (Industrial, Scientific and Medical) radio band and are based on Wi-Fi (eg., [6-8]), Bluetooth (eg., [9-11]) or ZigBee (eg., [12-14]) technologies. They use different methods for deriving the location information. For instance, location fingerprinting [1] is also a widely applied technique besides triangulation. In this case, signal fingerprints are collected in advance at a number of positions in the given area and later compared to the actual measurements. The location belonging to the best fit is selected as the position estimate. Unfortunately, none of these works investigate in a systematic way how to place the reference points optimally. Rather, they either use a pre-established infrastructure, or the placement is based on some heuristics or experimental results.

Considering the problem of optimal reference point placement, only minimum literature has been published so far to the best of our knowledge. The location optimization of Wi-Fi access points (AP) for fingerprint based positioning is a similar issue which has been investigated in some recent works. Baala et al. in [15] showed via measurements that the number and placement of the APs can have substantial impact on the position accuracy. But the authors do not propose a systematic way for finding an optimal AP deployment, rather the results are based on experiments. Zhao et al. in [16] proposed an AP location optimization method based on the Differential Evolution algorithm. In this method, the Euclidean distance of the RSS array between all the sampling points is maximized, by which the positioning accuracy can be improved. Unfortunately, the model does not take into account the effect of walls, doors and other obstacles. He at al. in [17] proposed a rapid and optimal AP deployment scheme based on genetic algorithm, which maximizes the signal space Euclidean distance between the APs. The simulation results pointed out that "the more the better" rule does not necessarily hold, though the number of APs usually increases with the size of the target area. Similarly to the previous work, the authors used a simple signal propagation approach and did not consider the attenuation effects of the indoor environment. Fang and Lin in [18] presented a framework for linking the placement of APs and the positioning performance. Their algorithm aims at choosing a proper set of APs' locations so that the signal is maximized and the noise is minimized simultaneously. The location system is developed in a real-world environment collecting realistic measurements. However, collecting and comparing the measurement results of the different AP setups can be a cumbersome task especially in a large area to be covered.

OptiRef is a simulation based method and can complement the measurement based approaches by considerably reducing the reasonable reference point setups to be considered. In this sense, the outcome of OptiRef is a good starting point for further, real environment investigations. For example, one can deploy a setup resulted by OptiRef, create the signal map of the territory by measurements, and refine the placement of the reference points based on the measured signal map. 


\section{Reference Point Placement}

The triangulation method is a commonly used technique for positioning purposes in wireless environment. However, it demands the fulfillment of some basic requirements. Thus, the indispensable condition for triangulation is to receive the signal of at least three reference points everywhere within the given indoor area.

\subsection{Optimization of Reference Point Setup}

Finding the optimal number and positions of the reference points, which still satisfies the condition above, in real environment is a challenging task for analytical methods, because the propagation characteristics of wireless signals are too complex to be realistically modeled. Nevertheless, in order to find a setup with minimum number of reference sensors an obvious approach is to analyze and compare all the possible setups. Unfortunately, in real world this process is almost impossible to be accomplished, therefore simulations and optimization methods are to be used.

Actually, the number of reference sensor position combinations is infinite because the territory, where the sensors can be deployed, is continuous and contains infinite number of points available for sensor deployment. To handle this problem, we assume that the reference sensors can be located only in discrete points of the territory map. If the density of these points is high enough the original situation can be approximated well. For example, if we consider a $106 \mathrm{~m} \times 102 \mathrm{~m}$ indoor territory (eg., a parking garage) where the sensors can be placed into the junctions of a grid with $10 \mathrm{~cm}$ grid distance, then the number of possible reference point locations is $1,081,200$. Figure 1 illustrates this scenario but showing a grid with around $12 \mathrm{~m}$ grid distance for better visibility (the thick blue lines on the map represent walls). Of course, in a real environment it can happen that not all the grid points are valid locations for sensor deployment, and these points can be eliminated from the investigations.

Unfortunately, analyzing all the possible reference sensor setups with a brute force algorithm cannot be accomplished due to the huge number of location setup combinations. In the previous example, $2^{1081200}$ different sensor location setups exist that cannot be processed in acceptable time. To solve this problem, alternative solutions are required. 


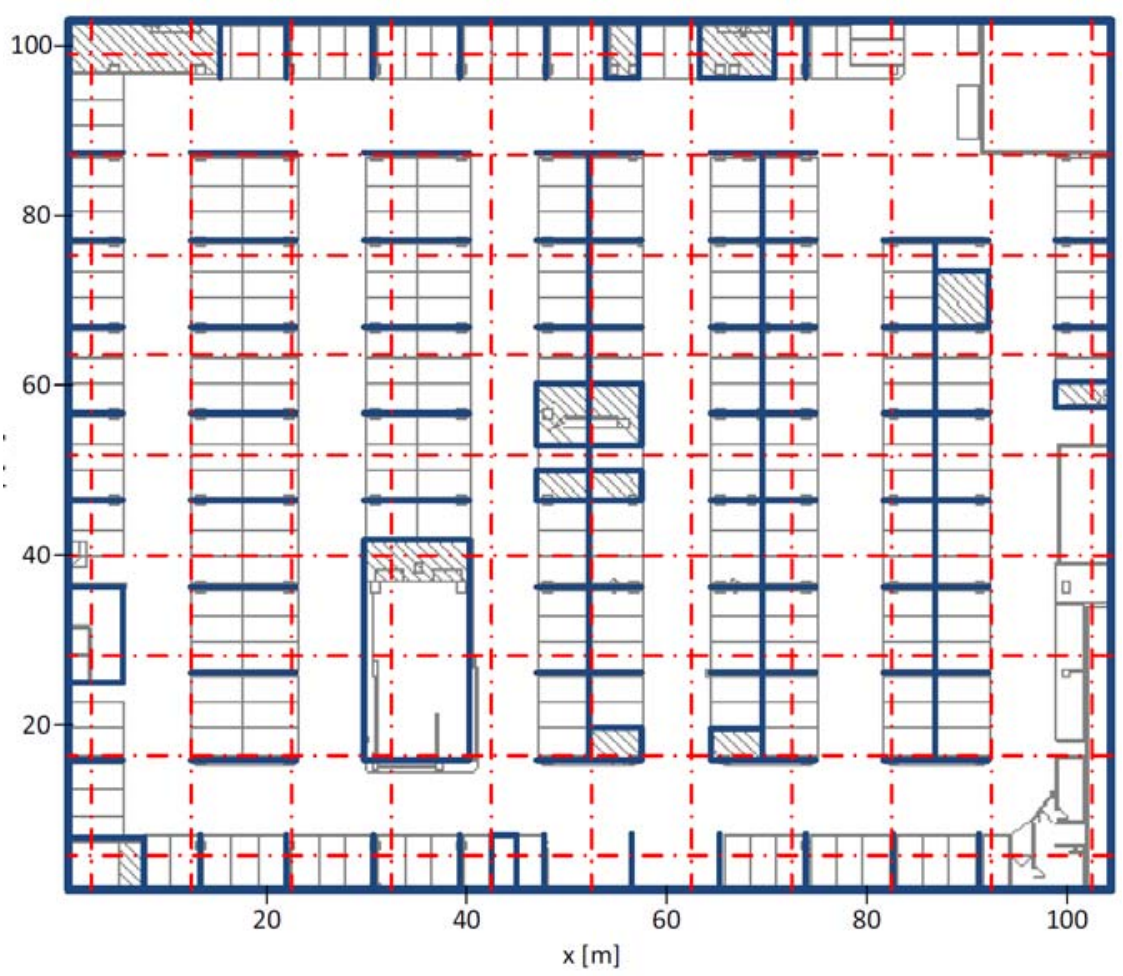

Figure 1

Territory map with a grid representing the possible reference sensor locations

\subsection{OptiRef Algorithm}

We propose OptiRef, our top-down reference point placement algorithm to find the optimal reference sensor location setup(s).

We start with a simple method. The initial step is to place a reference sensor in every discrete grid junction point of the territory map and compute the coverage area of each sensor using a wireless signal propagation model. In real environment, this can be almost any point of the continuous space, but we consider only discrete points with high density, equals to the map resolution in our simulations, in order to make the calculations possible. The next step is to determine the number of perceived reference sensors, using the previously computed coverage maps, in each point of the territory where the visiting node can be located, and thus to verify the fulfillment of the criterion. If there is no point on the map where the number of perceived reference sensors is less than three, than one sensor can be removed randomly. The next step is to check the criterion again. 
If the number of perceived sensors still fulfills it in each point of the territory, then another sensor can be removed randomly and so on, otherwise the algorithm stops. This simple method provides a solution, but in most of the cases not an optimal one.

This method can be modeled in a tree graph, where the states represent by binary numbers all the possible reference point combinations. After serializing the grid (creating from the 2D grid a 1D sequence by writing down the rows of the grid one after the other) the binary number determines which sensor is part of the given setup. The root state (every digit is a 1) is the initial setup containing in every grid junction point a reference sensor. The states one level below represent the setups where a reference sensor is deployed in all but one grid junction point, or from a different viewpoint one sensor is removed compared to the parent state, and so on. For instance, 1011..1 means that the reference sensor in the second position is removed compared to the initial setup. Figure 2 illustrates this graph containing the possible reference point combinations. Note, that the Hamming distance between the neighboring levels is one, and the graph consists of $n$ levels if the number of possible reference point locations (grid junction points) is $n$.

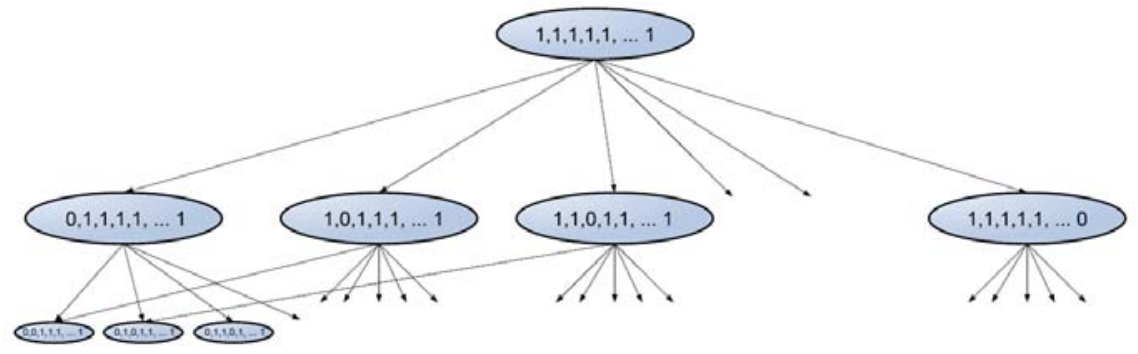

Figure 2

Graph representation of the possible reference sensor setups

With the graph representation we can originate our task in a graph theory problem. Thus, our goal is to find the state with the longest distance from the root, in which state the three perceivable reference sensors criterion still holds. On each level of the tree, the number of removed reference points is the same, therefore the deeper we are in the tree the less reference points are needed to cover the served territory. However, this "longest path" task is an NP-complete problem in graph theory [19].

Numerous heuristic optimization algorithms were developed to find the global optimum for such NP-complete problems, like hill climbing, swarm intelligence, integer linear programming, simulated annealing, etc. For our case, we propose simulated annealing to approximate the optimal reference point setup. Simulated annealing is a generic probabilistic algorithm for global optimization [19]. It tries to locate a good approximation of a given function's global optimum in a large search space even for NP-complete problems. 
OptiRef extends the above-mentioned simple reference point placement method with the simulated annealing algorithm. Hence, a previously removed reference point can be put back again with some probability. This probability is given in Equation 1:

$$
\exp (-\Delta E / T)
$$

where $\Delta E$ stands for the cost function difference of the two neighboring reference sensor setups in question and parameter $T$ is called temperature. The cost function, in our case, is the number of reference points in the given graph state. $T$ is the sum of the number of perceived reference sensors for each position on the territory map. This number is decreasing as more and more sensors are removed (we are deeper in the graph), that can be interpreted as "cooling" in the context of simulated annealing. The possibility of putting a previously removed reference sensor back prevents the method from being stuck in a local minimum that is worse than the global one. Algorithm 1 shows the pseudo-code of the OptiRef algorithm's main steps.

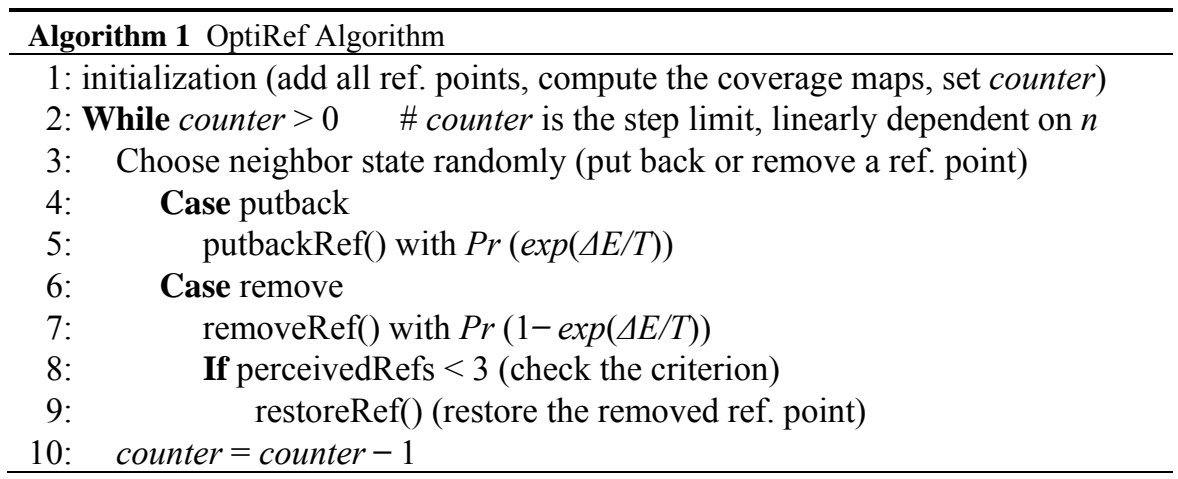

The time required to get an appropriate reference point setup is an important issue that is affected by the complexity of the algorithm used. To find the global optimum with a brute force method all the possible reference point setups must be investigated. Thus, it has an $O\left(2^{n}\right)$ complexity, where $n$ is the number of possible reference point locations. In case of the OptiRef algorithm, a step limit, linearly dependent on $n$, is used to determine how many times reference point can be removed or put back which limits the running time of the algorithm, too. Hence, our method, having $O(n)$ complexity, is able to find a good approximation of the global optimum in real time showing linear run-time behavior. 


\section{Evaluation}

The proposed OptiRef algorithm was evaluated via simulations. The simulation environment, the obtained results and some concluding remarks are presented in the following.

\subsection{Simulation Environment}

We have used the MATLAB [4] environment to develop our simulation tool. In this tool, we implemented first the ITU indoor wireless signal propagation model [5] that we applied in our simulations. The site-general ITU indoor model predicts the propagation path loss according to Equation 2:

$$
L=20 \cdot \log (f)+N \cdot \log (d)+L f(k)-28 d B,
$$

where $L$ is the total path loss $(\mathrm{dB}), f$ is the frequency $(\mathrm{MHz}), N$ is the distance power loss coefficient, $d$ is the distance between the transmitter and the receiver $(\mathrm{m}), L f(k)$ is the floor penetration loss factor and $k$ is the number of floors between the transmitter and the receiver.

In our investigations, we used only a single floor environment, a sector on one level of a parking garage. However, the ITU indoor signal propagation model is applicable also in a multi floor environment setting the $L f(k)$ parameter accordingly, that can be useful for the investigation of other indoor positioning based applications, too.

We selected the model and the simulation parameters according to the recommendations in [5] taking into account the properties of the simulated scenario (a parking garage). These parameters are: frequency, $N, L f(k)$, transmitter antenna gain, receiver antenna gain and transmitted power. We set the default values of these parameters to $2.4 \mathrm{GHz}, 27,10,5 \mathrm{~dB}, 2 \mathrm{~dB}$, and $30 \mathrm{~mW}$, respectively.

In wireless positioning systems, the RSS determines the range within the positioning service can be provided. If the signal is weak and the RSS is too low, the reference sensor is not perceived by the visiting terminal and cannot be used for positioning purposes. Thus, in order to determine the reference point coverage area we have introduced the terminal sensitivity parameter $(-80 \mathrm{~dB})$. If the received signal strength is lower than the terminal sensitivity, the terminal is out of the reference point's range.

In the simulator, we implemented not just our simulated annealing based OptiRef algorithm, but also a brute force method. In cases, when the number of possible reference point positions is not too high the brute force method is a better choice providing always the global optimal solution. However, due to the NPcompleteness of the problem finding the optimal reference sensor topology with 
the brute force method in real scenarios is usually not possible. In OptiRef, we set the step limit counter value as 10 times the number of the possible reference point positions giving the possibility to the algorithm not to get stuck in a local optimum. Table 1 summarizes the parameter settings we used in our simulations.

Table 1

Simulation parameter settings

\begin{tabular}{|c|c|c|}
\hline \multicolumn{3}{|c|}{ Wireless signal propagation model } \\
\hline \multicolumn{3}{|c|}{ ITU indoor } \\
\hline Frequency & $N$ & $L f(k)$ \\
\hline $2.4 \mathrm{GHz}$ & 27 & 10 \\
\hline Tx antenna gain & Rx antenna gain & Tx power \\
\hline $5 \mathrm{~dB}$ & $2 \mathrm{~dB}$ & $\mathrm{~mW}$ \\
\hline \multicolumn{3}{|c|}{ Terminal sensitivity } \\
\hline \multicolumn{3}{|c|}{ Step limit of OptiRef (counter) } \\
\hline \multicolumn{3}{|c|}{$10 \times$ no. of the possible reference point positions } \\
\hline
\end{tabular}

Moreover, the simulated area (map) has to be loaded at the beginning of the simulation process into our simulation tool. A .bmp image file can be used to determine the simulated environment by defining the rooms, walls, pillars, etc.

We ran the simulations on a Dell Inspiron 14z laptop equipped with Intel Core i5 2430M CPU@2.4 GHz, 4 GB RAM and MS Windows7 (64bit) operating system.

\subsection{Simulation Results}

In order to analyze the performance of our method we ran a number of simulations. Hence, we compared the simulation running times of OptiRef and the brute force algorithm first. Then we investigated the outcomes of OptiRef after several runs in case of a given scenario to see how close the resulted reference point numbers are to each other and how the simulation run-times vary. Next, we took a closer look at one simulation outcome and examined the coverage map of the resulted reference point setup. And finally, we investigated how the resulted reference point numbers change if the initial criterion is modified.

\subsubsection{OptiRef vs. Brute Force}

In the first simulation round, we compared our OptiRef algorithm to the brute force method and investigated their limitations. We used a simple indoor map with no walls and obstacles. 
In Figure 3, the average simulation run-times are shown, which were measured in function of the number of possible reference point positions and iterated 10 times for each setup, together with their $95 \%$ confidence intervals. We increased the number of possible reference point locations to see the scalability of OptiRef. In case of the brute force algorithm, we could not complete all of these simulations because they would have required too much time. Thus, we could investigate the required run-time of the brute force method only up to 20 possible reference point positions.

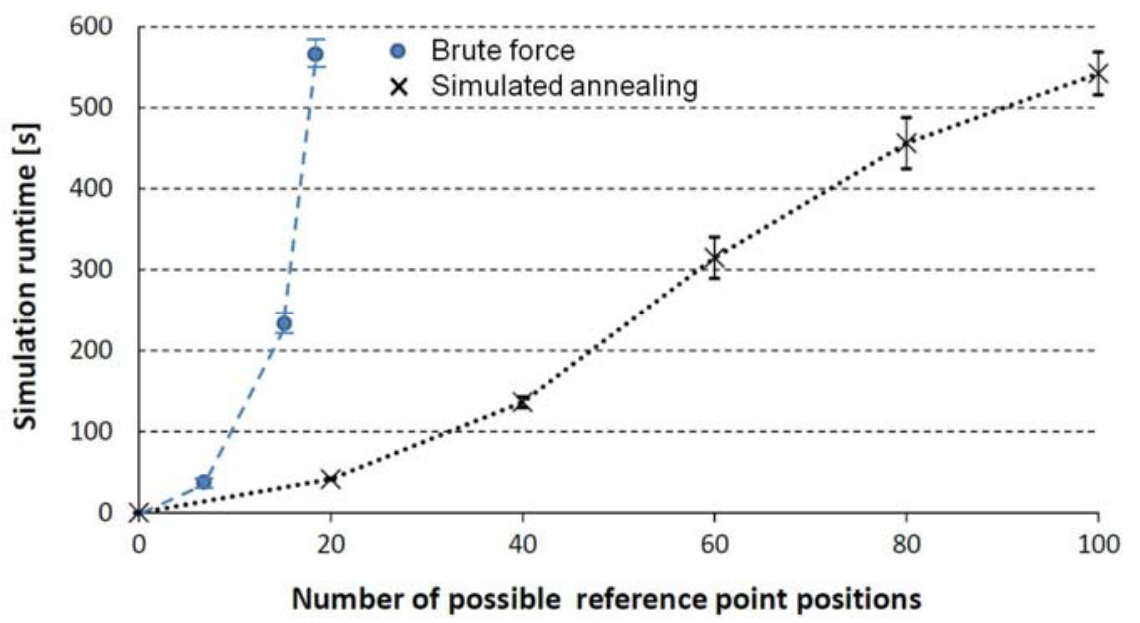

Figure 3

Simulation time vs. number of possible reference point positions in case of OptiRef and the brute force algorithm

The obtained results support that OptiRef scales well with the number of possible reference point positions showing a linear run-time increase. On the contrary, in case of the brute force algorithm the simulation run-time increases exponentially, as expected. Thus, in bigger scenarios with hundreds or thousands possible reference sensor locations the brute force method is practically unusable.

\subsubsection{Performance of OptiRef}

In the second round, we investigated further our OptiRef algorithm in a $106 \mathrm{~m} \times$ $102 \mathrm{~m}$ territory, where the grid distance was around $12 \mathrm{~m}$. The used territory map is illustrated in Figure 1. In our case, the number of possible reference point locations was 78 meaning $2^{78}$ different sensor topology setups. Of course, the grid density can be increased for the price of increased simulation run-time.

We repeated the simulation 10 times investigating the simulation run-time, consisting of the coverage map computation and the simulated annealing algorithm, and the resulted number of required reference points. The results are 
collected in Table 2. The mean simulation run-time was 457.46 seconds, from which the time needed for the coverage map calculations is notable $(456.87 \mathrm{sec})$, while only 0.59 seconds were required for the simulated annealing algorithm. This result is in line with our expectations. The coverage map calculation is time consuming especially when we have a huge number of reference sensors. On the other hand, the simulated annealing algorithm requires only some comparisons and a more or less random decision which can be carried out quickly.

Table 2

Mean run-time of 10 OptiRef simulations using the same scenario

\begin{tabular}{|c|c|c|c|c|}
\hline & $\begin{array}{c}\text { Total sim. } \\
\text { time [sec] }\end{array}$ & $\begin{array}{c}\text { Coverage map } \\
\text { calc. time [sec] }\end{array}$ & $\begin{array}{c}\text { Sim. annealing } \\
\text { alg. time [sec] }\end{array}$ & $\begin{array}{c}\text { No. of required } \\
\text { ref. points }\end{array}$ \\
\hline $\begin{array}{c}\text { Mean \& } \\
\text { std. }\end{array}$ & $457.46 \pm 5.22$ & $456.87 \pm 5.15$ & $0.59 \pm 0.09$ & $30.2 \pm 1.8$ \\
\hline
\end{tabular}

Note, that simulated annealing randomly chooses the neighbor states in the graph, therefore in case of several optimal solutions the resulted reference sensor setup can be different in consecutive simulation runs, even if the input parameters are the same. An output of the simulation is presented in Figure 4 where the selected reference point locations and RSS values are illustrated. The colors represent the highest RSS value in the given point of the territory which usually, but not necessarily, belongs to the closest reference sensor in the vicinity of the measurement.

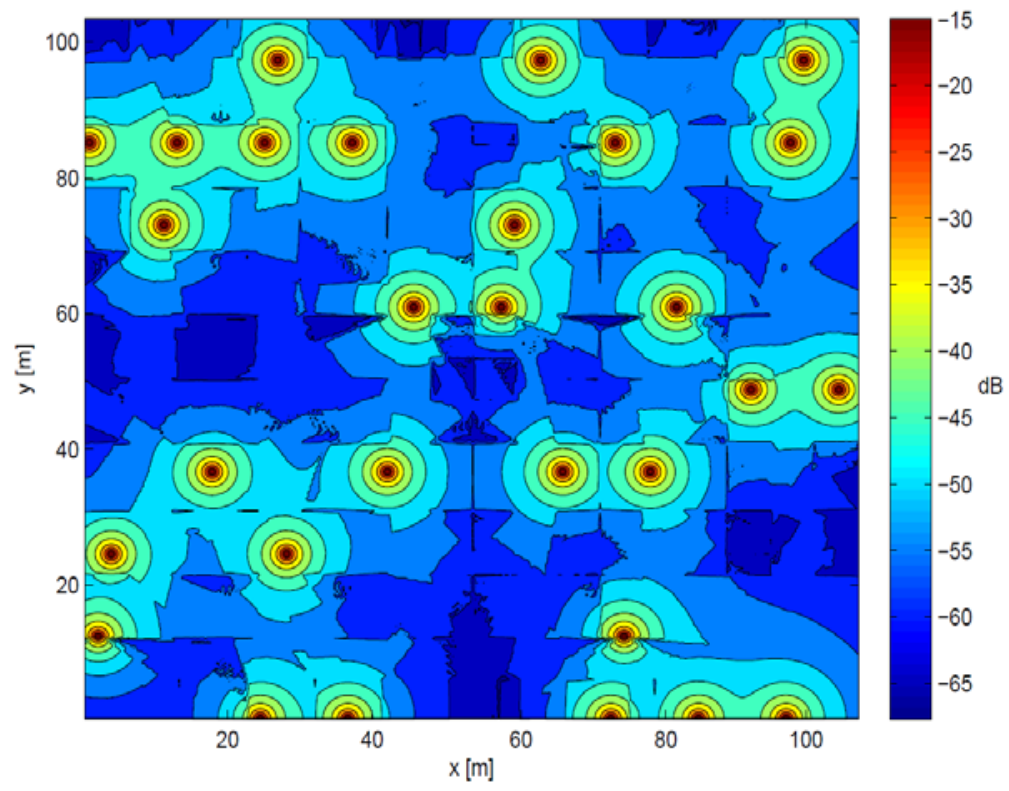

Figure 4

Selected reference point locations and RSS values using OptiRef 
29 reference points were the minimum still enough to cover the territory by satisfying the perceivability criterion. However, we can never be sure that we found a global optimal solution (or reached only a local optimum), but repeating the simulation increases its probability. In normal scenarios, a simulation running time takes some hundred (see Table 2) or probably some thousand seconds (depending on the grid density) on a usual computer, so iterating the simulation a couple of hundred times should not cause any problem today.

Analyzing several simulation outcomes we can notice that the algorithm locates the reference points in the border areas in most of the cases and only few sensors are placed in the center. The reason is that the perceivability criterion is censorious at the boundaries of the map; hence more sensors must be deployed at the edges of the territory.

\subsubsection{Coverage Map}

In the third round, we examined the reference sensor coverage density achieved by our OptiRef algorithm. The developed simulation tool makes it possible to analyze the number of perceived reference points in the served territory. If the RSS is higher than the terminal sensitivity $(-80 \mathrm{~dB})$, the reference point is assumed to be available for positioning purposes. Figure 5 summarizes the number of available reference sensors considering all the measurement points. Figure 6 illustrates the same on the territory map (the different numbers are represented by different colors) in case of a given reference sensor setup.

We can see, that some parts of the territory, mainly the center areas, are covered by 10-12 reference sensors, while the terminals visiting the border areas can receive the signal of only few sensors. Nevertheless, our perceivability criterion still holds everywhere in the territory.

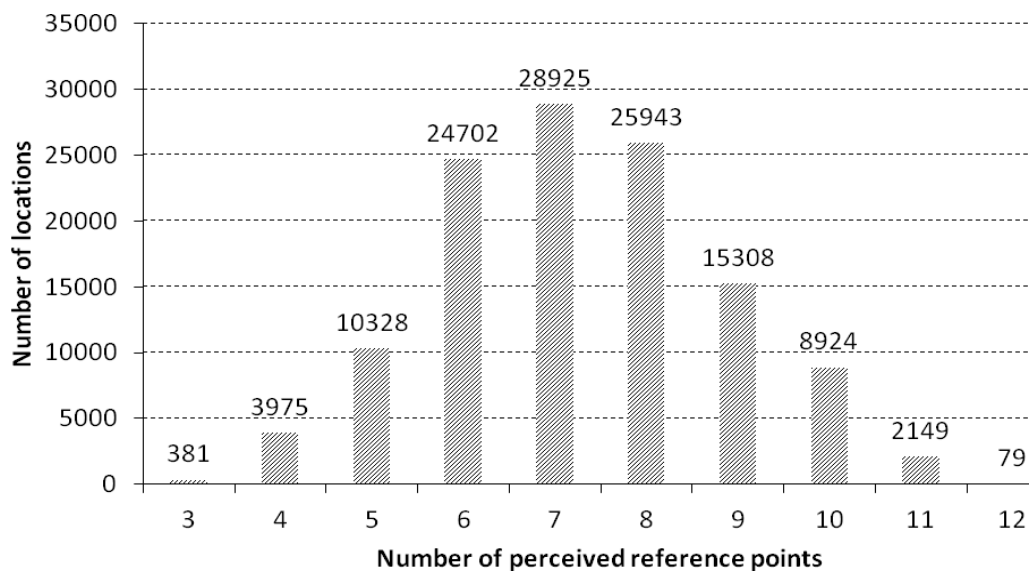

Figure 5

Number of reference points available for positioning 


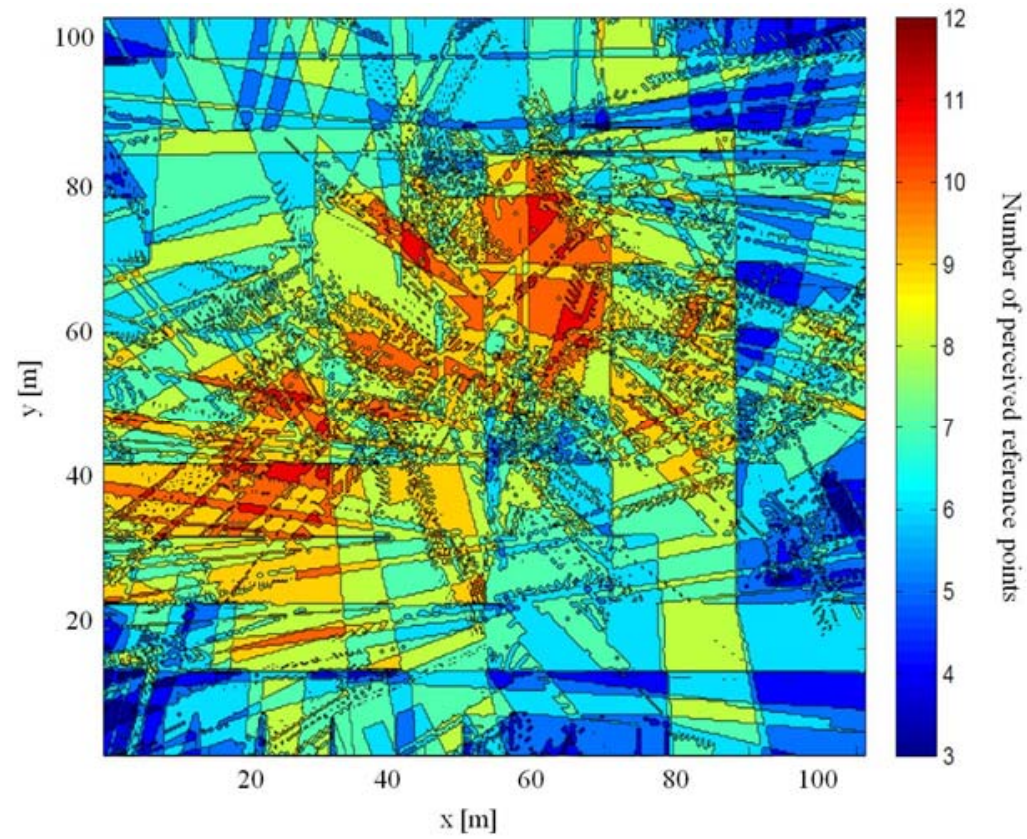

Figure 6

Map representation of the number of reference points available for positioning

\subsubsection{Impact of the Criterion Change}

In the forth round, we investigated the impact of changing the perceivability criterion. The perceivability of at least three reference sensors is a strict minimum requirement. However, by increasing the number of available reference sensors in a given point of the map the position estimation accuracy can be improved. We have analyzed the total number of required reference points if the minimum criterion of perceivable sensors is increased. The simulations were iterated 20 times and the results are shown in Figure 7 using the same territory map with the same 78 possible reference point locations, as in the previous experiments.

As it is expected, the number of required reference points for the positioning system is increasing if more than three sensors must be perceived in any point of the territory. As we noted before, the location and the number of reference points returned by OptiRef may vary due to the randomness of the simulated annealing algorithm. In Figure 7 the average, the minimum and the maximum number of required reference points are depicted using the same simulation setup. Although the differences are not significant, it is recommended to iterate the algorithm in order to find a sensor topology close to the global optimum. 


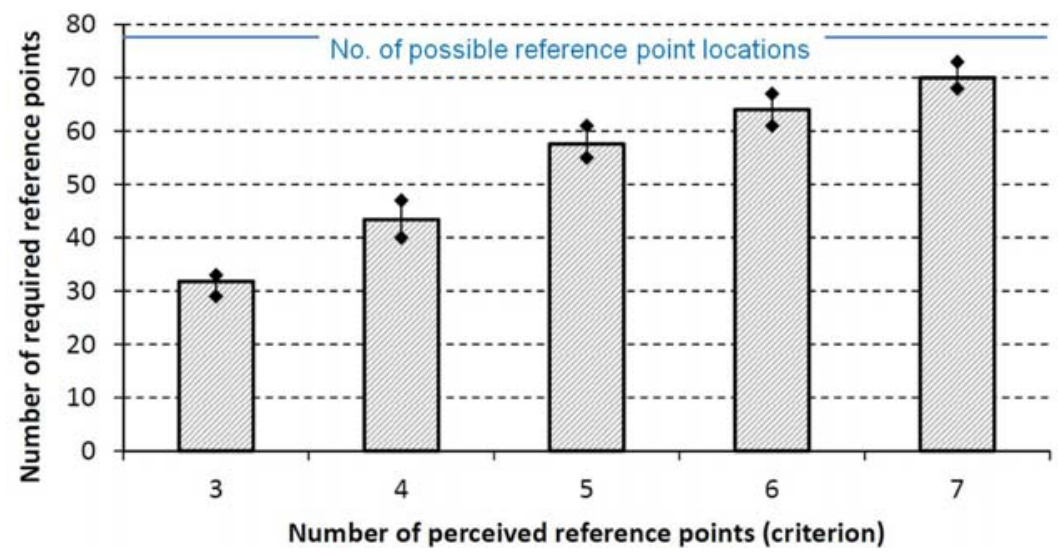

Figure 7

Number of reference points required to fulfill the perceivability criterion

\subsubsection{Discussion}

From the evaluation results we can see that OptiRef provides a solution for a given scenario within an acceptable time, if the number of possible reference point locations (grid density) is not too big (falls in the range of some hundreds). However, there is no guarantee that the resulted solution is a global optimal one. Hence, it is recommended to iterate the simulation to increase the probability of finding a global optimum. OptiRef is scalable, the simulation running time is linearly dependent on $n$, the number of grid points (potential reference sensors) in the given area. Moreover, OptiRef is flexible and can easily handle if the perceivability criterion changes. The simulation run-time does not increase in this case either because the time consuming part (coverage map calculations) does not change and it is performed only once in the initial phase.

An interesting issue is to think about how close to reality the simulation results are. In our case, it depends on the used wireless signal propagation model to a great extent. We ran also some simulations using a modified version of the freespace propagation model [5] and we introduced some wall attenuation (4dB) in computing the path loss. With this model we got slightly different results, the minimal reference point setup contained 25 sensors in case of the same scenario. Nevertheless, the signal propagation model can be easily replaced and using more realistic model results in solution closer to the real environment optimum.

\section{Conclusions}

In this paper, we investigated the issue of ZigBee reference sensor placement optimization for indoor positioning. Hence, we examined how to place the reference points to perceive the signal of at least three reference sensors everywhere within the given indoor territory, but keep the number of deployed 
sensors as low as possible. We proposed OptiRef, a simulated annealing based method to find a good approximation of the optimal solution. OptiRef has $O(n)$ complexity and shows linear run-time behavior. Furthermore, we have developed a simulation tool in MATLAB environment for the given problem. We used this tool to implement OptiRef together with the ITU indoor wireless signal propagation model and to investigate the algorithm's behavior.

By minimizing the amount of required reference points the cost of deployment and operation expenses can be reduced, while at the same time still providing an efficient positioning system. OptiRef and the developed simulation tool are generic and they can be useful in planning radio-based positioning systems not just focusing on ZigBee technology. The simulator is adaptable to different wireless technologies by adjusting the signal propagation parameters or even by replacing the propagation model. The reference point setup(s) resulted by OptiRef can be considered as a good starting point for real environment design.

As future work, we plan to further investigate the performance and limitations of OptiRef. We will collect real measurements and compare them with our simulation results. Moreover, in some scenarios it can be desirable to deploy such a reference point setup in which the sensors form a connected wireless sensor network. We plan to extend our algorithm to satisfy this condition, too.

\section{Acknowledgement}

The publication was supported by the EITKIC 12-1-2012-0001 project, which is supported by the Hungarian Government, managed by the National Development Agency, financed by the Research and Technology Innovation Fund and was performed in cooperation with the EIT ICT Labs Budapest Associate Partner Group (www.ictlabs.elte.hu). Károly Farkas has been partially supported by the Hungarian Academy of Sciences through the Bolyai János Research Fellowship. The author would like to acknowledge the support and help of Árpád Huszák and Győző Gódor in this work.

\section{References}

[1] H. Liu, H. Darabi, P. Banerjee, and J. Liu, "Survey of Wireless Indoor Positioning Techniques and Systems," IEEE Transactions on Systems, Man, and Cybernetics, Part C: Applications and Review, Vol. 37, No. 6, pp. 1067-1080, Nov. 2007

[2] Y. Gu, A. Lo, and I. Niemegeers, "A Survey of Indoor Positioning Systems for Wireless Personal Networks," IEEE Communications Surveys and Tutorials, Vol. 11, No. 1, pp. 13-32, 2009

[3] H. Koyuncu and S. H. Yang, "A Survey of Indoor Positioning and Object Locating Systems," International Journal of Computer Science and Network Security, Vol. 10, No. 5, pp. 121-128, May 2010 
[4] MathWorks, "MATLAB and Simulink for Technical Computing," 2012, www.mathworks.com

[5] J. S. Seybold, Introduction to RF Propagation. John Wiley \& Sons, Inc., Sep. 2005

[6] P. Bahl and V. N. Padmanabhan, "RADAR: An In-Building RF-based User Location and Tracking System," in Proceedings of the $19^{\text {th }}$ IEEE International Conference on Computer Communications (INFOCOM 2000) Vol. 2, Tel Aviv, Israel, Mar. 2000, pp. 775-784

[7] M. Youssef, A. Agrawala, and A. Udaya Shankar, "WLAN Location Determination via Clustering and Probability Distributions," in Proceedings of the First IEEE International Conference on Pervasive Computing and Communications (PerCom 2003) Dallas-Fort Worth, TX, USA, Mar. 2003, pp. $143-150$

[8] T. King, S. Kopf, T. Haenselmann, C. Lubberger, and W. Effelsberg, "COMPASS: A Probabilistic Indoor Positioning System Based on 802.11 and Digital Compasses," in Proceedings of the First ACM International Workshop on Wireless Network Testbeds, Experimental evaluation and CHaracterization (WiNTECH 2006) Los Angeles, CA, USA, Sep. 2006, pp. $34-40$

[9] A. Genco, "Three Step Bluetooth Positioning," in Proceedings of the First International Conference on Location- and Context-Awareness (LoCA'05) Oberpfaffenhofen, Germany, May 2005, pp. 52-62

[10] M. Rodriguez, J. P. Pece, and C. J. Escudero, "In-Building Location Using Bluetooth," in Proceedings of the International Workshop on Wireless Adhoc Networks (IWWAN 2005) London, UK, May 2005

[11] S. Kawakubo, A. Chansavang, S. Tanaka, T. Iwasaki, K. Sasaki, T. Hirota, H. Hosaka and H. Ando, "Wireless Network System for Indoor Human Positioning," in Proceedings of the $1^{\text {st }}$ Inernational Symposium on Wireless Pervasive Computing (ISWPC 2006), Phuket, Thailand, Jan. 2006, pp. 1-6

[12] O. Hernandez, V. Jain, S. Chakravarty and P. Bhargava, "Position Location Monitoring - Using IEEE 802.15.4/ZigBee technology," Beyond Bits, Vol. 4, pp. 67-73, Summer 2009

[13] K. Maneerat and C. Prommak, "On the Analysis of Localization Accuracy of Wireless Indoor Positioning Systems using Cramer's Rule," World Academy of Science, Engineering and Technology (WASET) - Online Special Issue, Vol. 60, pp. 202-207, Apr. 2011 
[14] S.-H. Fang, C.-H. Wang, T.-Y. Huang, C.-H. Yang and Y.-S. Chen, "An Enhanced ZigBee Indoor Positioning System with an Ensemble Approach," IEEE Communications Letters, Vol. 16, No. 6, pp. 564-567, Apr. 2012

[15] O. Baala, Y. Zheng and A. Caminada, "The Impact of AP Placement in WLANbased Indoor Positioning System," in Proceedings of the Eighth International Conference on Networks (ICN'09) Cancun, Mexico, Mar. 2009, pp. 12-17

[16] Y. Zhao, H. Zhou and M. Li, "Indoor Access Points Location Optimization Using Differential Evolution," in Proceedings of the International Conference on Computer Science and Software Engineering (CSSE 2008), Vol. 1, Wuhan, China, Dec. 2008, pp. 382-385

[17] Y. He, W. Meng, L. Ma and Z. Deng, "Rapid Deployment of APs in WLAN Indoor Positioning System," in Proceedings of the 6th International ICST Conference on Communications and Networking in China (CHINACOM 2011) Harbin, China, Aug. 2011, pp. 268-273

[18] S.-H. Fang and T.-N. Lin, "A Novel Access Point Placement Approach for WLANBased Location Systems," in Proceedings of the IEEE Wireless Communications and Networking Conference (WCNC 2010) Sydney, Australia, Apr. 2010, pp. 1-4

[19] D. Karger, R. Motwani and G. Ramkumar, "On Approximating the Longest Path in a Graph,” Algorithmica, Vol. 18, No. 1, pp. 82-98, 1997 